Ann. Geophysicae 16, 775-786 (1998) @ C EGS - Springer-Verlag 1998

\title{
Observations of substorm fine structure
}

\author{
L. L. Lazutin ${ }^{1}$, R. Rasinkangas ${ }^{2}$, T. V. Kozelova ${ }^{1}$, A. Korth ${ }^{3}$, H. Singer ${ }^{4}$, G. Reeves ${ }^{5}$, W. Riedler ${ }^{6}$, K. Torkar ${ }^{6}$, \\ B. B. Gvozdevsky ${ }^{1}$ \\ ${ }^{1}$ Polar Geophysical Institute, Russian Academy of Sciences, Apatity, Murmansk Region, 184200, Russia \\ 2 University of Oulu, Department of Physical Sciences, FIN-90570 Oulu, Finland \\ ${ }^{3}$ Max-Planck-Institut für Aeronomie, D-37191 Katlenburg-Lindau, Germany \\ ${ }^{4}$ Space Environment Center, NOAA, 325 Broadway, Boulder, CO 80303-3328, USA \\ ${ }^{5}$ Los Alamos National Laboratory, Los Alamos, NM 87545, USA \\ ${ }^{6}$ Institute of Space Research of the Austrian Academy of Sciences, A-8010 Graz, Austria
}

Received: 24 September 1997 / Revised: 9 January 1998 / Accepted: 22 January 1998

\begin{abstract}
Particle and magnetic field measurements on the CRRES satellite were used, together with geosynchronous satellites and ground-based observations, to investigate the fine structure of a magnetospheric substorm on February 9, 1991. Using the variations in the electron fluxes, the substorm activity was divided into several intensifications lasting about 3-15 minutes each. The two main features of the data were: (1) the intensifications showed internal fine structure in the time scale of about 2 minutes or less. We call these shorter periods activations. Energetic electrons and protons at the closest geosynchronous spacecraft (1990 095) were found to have comparable activation structure. (2) The energetic $(>69 \mathrm{keV})$ proton injections were delayed with respect to electron injections, and actually coincided in time with the end of the intensifications and partial returns to locally more stretched field line configuration. We propose that the energetic protons could be able to control the dynamics of the system locally be quenching the ongoing intensification and possibly preparing the final large-scale poleward movement of the activity. It was also shown that these protons originated from the same intensification as the preceeding electrons. Therefore, the substorm instability responsible for the intensifications could introduce a negative feedback loop into the system, creating the observed fine structure with the intensification time scales.
\end{abstract}

Key words. Magnetospheric Physics (Storms and substorms).

\section{Introduction}

The development of magnetospheric substorms on large temporal and spatial scales is well established. Dis-

Correspondence to: L. L. Lazutin turbed periods can be morphologically divided into intervals like the growth, expansion and recovery phases with corresponding signatures (Rostoker et al., 1980), while the physical processes involved can be divided into directly driven and loading-unloading processes (Rostoker et al., 1987). Many details, however, are still not understood. For example, the substorm definition by Rostoker et al. (1980) included the concept of "multiple substorm onsets", and many observational results verify that a poorly understood fine structure is an inherent feature of substorms (e.g., Sergeev et al., 1996). Because of this, Sergeev et al. (1996) postulated the 1-2 min impulsive dissipation events (IDE) as the elementary building blocks of the substorm expansion.

Numerous observations have indicated that the substorm fine structure could actually be divided into two classes, one with a duration of about $2 \mathrm{~min}$ (or less) and another with a duration of 3-15 min. In the following we will use terms "activations" and "intensifications", respectively, for these two fine structure time scales. For example, ground based investigations have shown that intensifications like auroral breakups (including pseudo-breakups; Koskinen et al., 1993) and westward travelling surges comprise shorter elementary activations (Murphree and Cogger, 1992; Kisabeth and Rostoker, 1974; Pytte et al., 1976; Nakamura et al., 1993). Similar features can be seen in practically all ground-based data sets, see, e.g. Bösinger et al. (1981), Yahnin et al. (1983), Sergeev et al. (1986a, b), Bösinger and Yahnin (1987), Murphree and Elphinstone (1988), Williams et al. (1990), and Shephard and Murphree (1990).

Substorm related particle injections (Anderson, 1965; Mauk and McIlwain, 1974; McIlwain, 1974) and other intensifications in the equatorial magnetosphere can also have internal fine structure. For example, Belian et al. (1984) showed multiple peaked proton injections at the geosynchronous orbit. In a case study based on CRRES data, Grande et al. (1992) reported on two injections of 10 min duration that exhibited internal fine structure, 
"injectionlets". The finer details of the substorm onset structure have also been studied (e.g. Lui et al., 1988, 1992; Ohtani et al., 1992; Rasinkangas et al., 1994; Maynard et al., 1996; Sergeev et al., 1998). For example, the spiky, activation time scale electric field structures observed by Maynard et al. (1996) were interpreted as radial oscillations of fluxtubes due to Alfvén waves associated with the substorm current wedge formation. However, similar spikes observed deep in the inner magnetosphere by Sergeev et al. (1998) were interpreted as fast magnetosonic waves radiated from the distant current disruption region. Finally, we note that even the high-speed flows in the plasma sheet show fine structure comparable with the time scales of both intensifications and activations (Angelopoulos et al., 1992).

In this study, we will present a case study of a substorm observed by the CRRES satellite. We show how the activity is characterized by fine structure at both intensification and activation time scales, and propose a scheme that could explain the former.

\section{Data}

The CRRES satellite was launched on July 25, 1990 into an orbit with a perigee height of $350 \mathrm{~km}$, an apogee of $35786 \mathrm{~km}$, and an inclination of $18^{\circ}$. During the substorm on orbit 484, February 9, 1991, CRRES approached the midnight sector of the auroral magnetosphere at $\mathrm{L} \sim 6.3, \mathrm{MLT} \sim 22.9$, magnetic latitude decreasing from $-4.4^{\circ}$ to $-6.7^{\circ}$ between 17 and $18 \mathrm{UT}$. Figure 1 shows a projection of the CRRES orbit into the
GSM xy-plane and the geographic footprints of the field lines through the spacecraft (calculated using T89 model, $K p \sim 3$ ). The locations of some important event characteristics, which will be described later, are marked along the orbit.

On CRRES, the electron proton wide-angle spectrometer (EPAS, also known as MEB; Korth et al., 1992) measured electrons with energies between 21.5 and $285 \mathrm{keV}$ and ions (with no mass resolution) between 37 and $3200 \mathrm{keV}$. In this study, data with $30 \mathrm{~s}$ (one spin) time resolution and 19 pitch angle (PA) bins have been used. The fluxgate magnetometer provided magnetic field measurements (Singer et al., 1992).

The LANL synchronous orbit particle analyzer (SOPA) data from the geosynchronous orbit was also used in the study. The instrument measures electrons in the energy range $50 \mathrm{keV}-1.5 \mathrm{MeV}$ and protons in the range $50 \mathrm{keV}-50 \mathrm{MeV}$. During the present event, 1990095 was located near the magnetic midnight, only 40-60 minutes MLT eastward of CRRES, while 1987 097 was located in the dusk sector (about 1730 MLT). Both satellites are also marked in Fig. 1. In addition, ground-based magnetometer data from Tromsø, Apatity, Dixon, Tixie and Cape Wellen were used to investigate the spatial extent of the substorm activity; pulsation magnetometer data from Yakutsk provided timings for the activity onsets; and all-sky camera (ASC) pictures from Kilpisjärvi made it possible to investigate auroral activity to the west of the CRRES meridian. The locations of these stations are plotted on Fig. 1.

Finally, the IMF and solar wind conditions were studied using the data from IMP-8, which was located at about $(34,2,-16) \mathrm{R}_{e}$ GSM during the event.

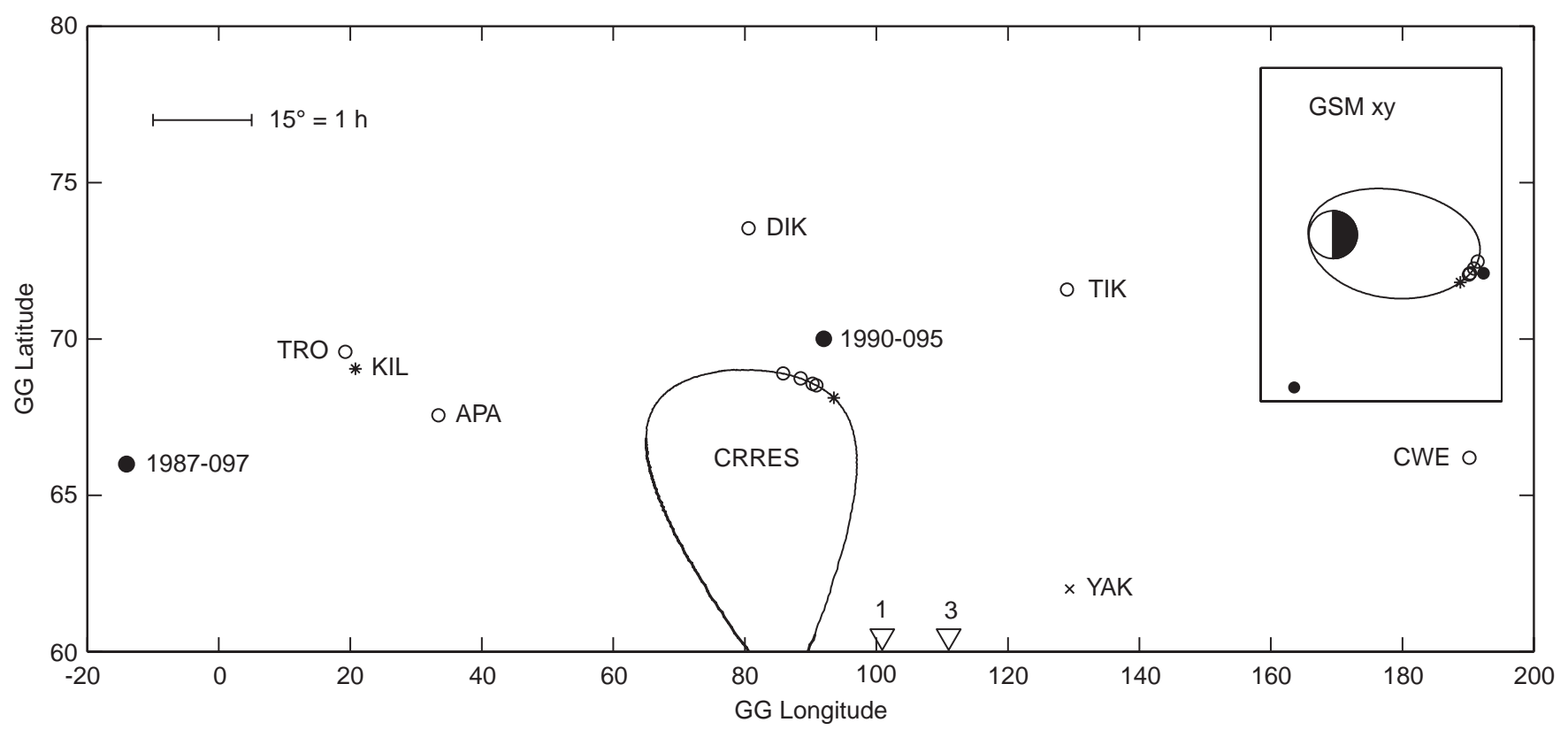

Fig. 1. Satellite footprints and ground stations in geographic coordinates on February 9, 1991. The substorm onset $(*)$ and the four intensifications (o) are marked along the CRRES orbit (see text for more details). The locations of the geosynchronous satellites $(\bullet)$ are shown for 1700 UT. Equatorial projection in GSM coordinates can be seen in the inset figure 


\section{Observations}

\subsection{Ground-based data}

The development of the substorm is outlined in Table 1, which lists the main features as seen from ground, geosynchronous orbit and CRRES. The ground-based magnetometer data ( $H$-component) from five auroral zone observatories are shown in Fig. 2. The MLT midnight times in UT hours are given for each station in the parenthesis, and marked along the curves with dots when they fall in the time period shown. The strong positive magnetic bay indicating increased eastward current started in Apatity at about 1610 UT. This growth phase was followed by a negative magnetic bay that started at about 1657 UT and contained several intensifications during its two-hour active phase. The substorm onset time is verified also from the Yakutsk data (not shown) displaying Pi2 onset at the same time (both timings are subject to \pm 1 min errors). The activity region extended azimuthally from at least Tromsø to Cape Wellen with several localized substructures. More careful analysis (including also the $Z$-component not shown here) indicates that the westward electrojet stayed south of Dixon until about 1740 UT, when the poleward expansion started. During this time the substorm reached also the Kilpisjärvi station, as seen from the

Table 1.

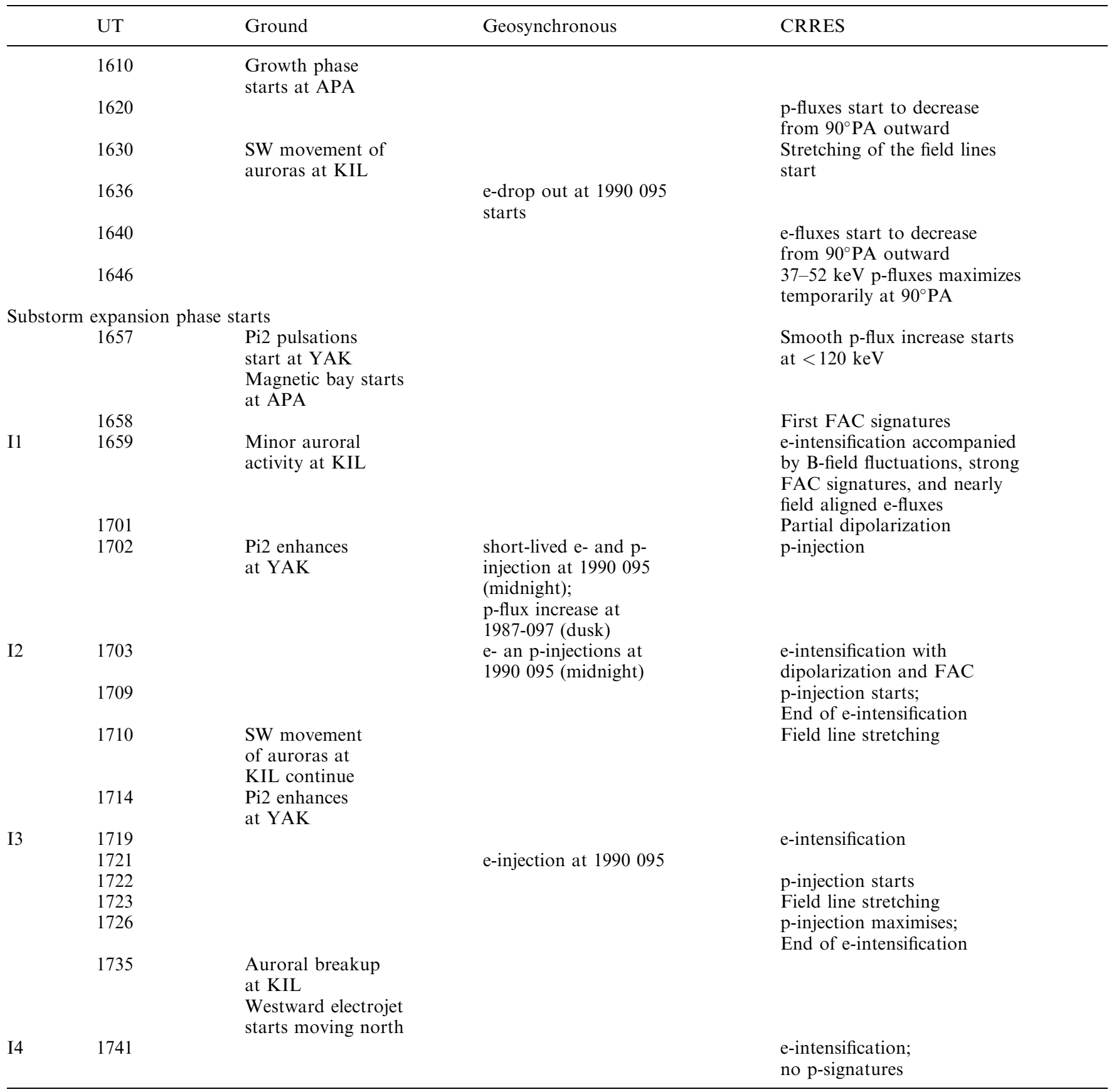




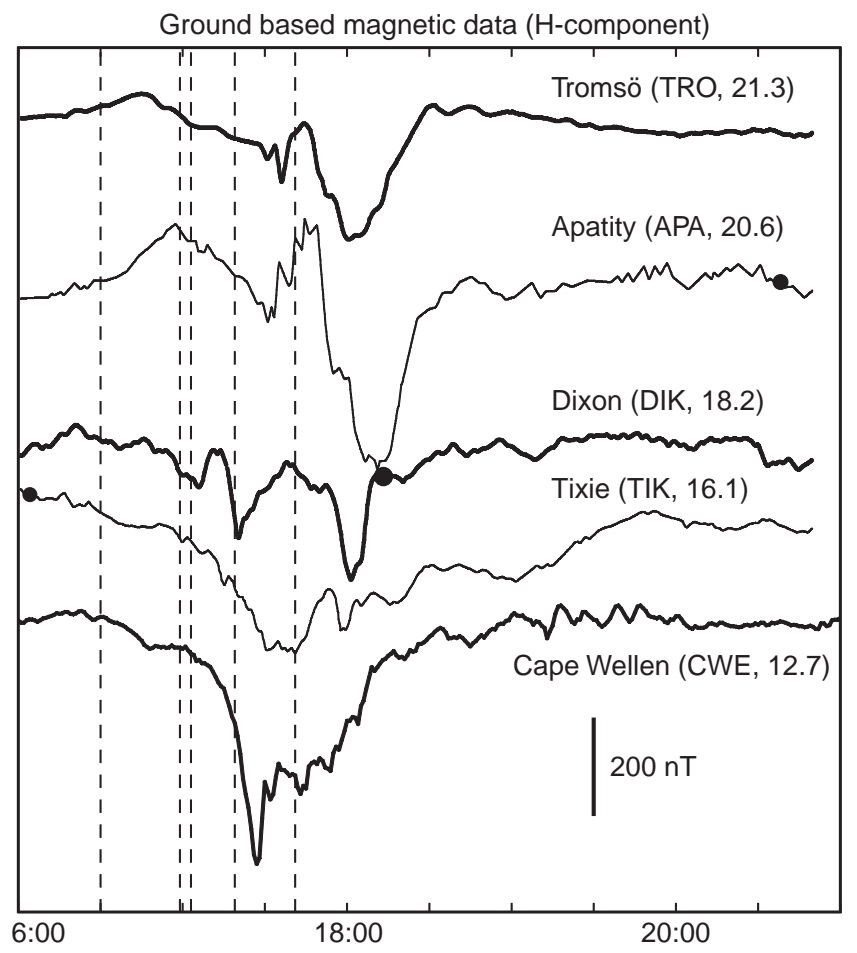

Fig. 2. $H$-component of magnetic field variations, registered by ground-based magnetometers in Tromsø, Apatity, Dixon, Tixie Bay and Cape Wellen on February 9, 1991. The growth phase onset and the four intensifications seen by CRRES and discussed in the text are marked as dashed vertical lines

ASC pictures (data not shown, see Table 1). The vertical lines in the figure will be discussed later.

\subsection{Overview of the satellite data}

Figure 3 shows an overview of the satellite data used in this study. The uppermost panel displays IMF $B_{z}$ component (SE) as measured by IMP-8. Although there are serious data gaps, it seems that the IMF had a strong southward component during most of the event. This period started at about 1606 UT (not shown here), and continued until about 1735 UT. In addition, there is a sharp decrease in the strength of the southward component at about 1651 UT, i.e., just before the substorm onset, and a short excursion to zero level shortly after 1700 UT. The solar wind parameters were quite constant, the velocity being about $420 \mathrm{~km} / \mathrm{s}$ (data not shown).

The rest of the panels in Fig. 3 show the magnetospheric data. The second panel shows the 1987097 protons, the next three panels the CRRES electron fluxes, proton fluxes (both at $90^{\circ} \mathrm{PA}$ ) and magnetic components, respectively, and the lowermost two panels the 1990095 electron and proton fluxes. The start of the substorm growth phase can be seen as stretching of the field lines at CRRES, i.e. decrease of $B_{z}$ starting at about 1630 UT (panel 5; note that Table 1 lists several other growth phase related signatures in the particle data, some of which are best seen in the colour-coded pitch angle distribution plots not shown here). At least four substorm related electron injections followed (I1-I4 in Table 1, marked as horizontal bars in panel 3), the first one being delayed from the first ground based onset signatures by a couple of minutes. The first two injections related to local magnetic field dipolarization and field-aligned currents ( $B_{y}$ variations in panel 5), and the last one correlate with the northward turning of the IMF $B_{z}$ and the final poleward expansion of the activity. Because of their durations, about 3, 7, 7, and $12 \mathrm{~min}$ respectively, we consider these injections to be intensifications as defined in the Introduction. The five vertical dashed lines in Fig. 3 mark the (CRRES) growth phase onset and the starting times of the intensifications. The same information is also marked along the CRRES orbit in Fig. 1, and as vertical dashed lines in Fig. 2. See Table 1 for corresponding UT times (note that a data gap just after 1740 UT makes it impossible to estimate exactly the start time for the last intensification, and that its exact duration is also difficult to define due to the very small flux variations that follow).

Of the geosynchronous satellites, the duskside 1987 097 showed the gross features of the substorm related injections as the protons drifted from the midnight sector toward it (panel 2). On the other hand, 1990 095, located very close to CRRES, showed details that resemble those in the CRRES data (panels 6 and 7 in Fig. 3). The substorm growth phase is seen as an electron flux drop out that recovered during the second CRRES intensification. Also the third intensification is clearly seen in the electron data with some delay.

To end this overview, we note that there are several reasons to consider the two first intensifications as separate events, although the first one is so short: (1) the electron spectra and pitch angle distributions at CRRES are different, as will be discussed later; (2) there is an additional $\mathrm{Pi} 2$ enhancement related closely with I2 (Table 1, the timing has an error of about one minute); (3) the 1987097 data shows a double peaked increase in the duskside proton flux a few minutes later (see the two vertical ticks in panel 2, Fig. 3); (4) the 1990095 electrons and protons behave differently for these two intensifications (panels 6 and 7, see also next subsection); and (5) the CRRES electric field data (not shown, personal communication with N. Maynard) displays a strong, separate duskward spike during each of the four intensifications.

We will first examine the fine structure within the intensifications (most clear during the second and third intensification; see Fig. 3, panel 3), and then discuss a repeating pattern that can be seen in the data, i.e., the fact that, in the first three intensifications, electron injection is followed by a proton injection that coincides in time with the end of the intensification (Table 1 and panel 4 in Fig. 3).

\subsection{Intensification characteristics}

Figures 4 and 5 display CRRES data from the intensification periods with a higher time resolution 


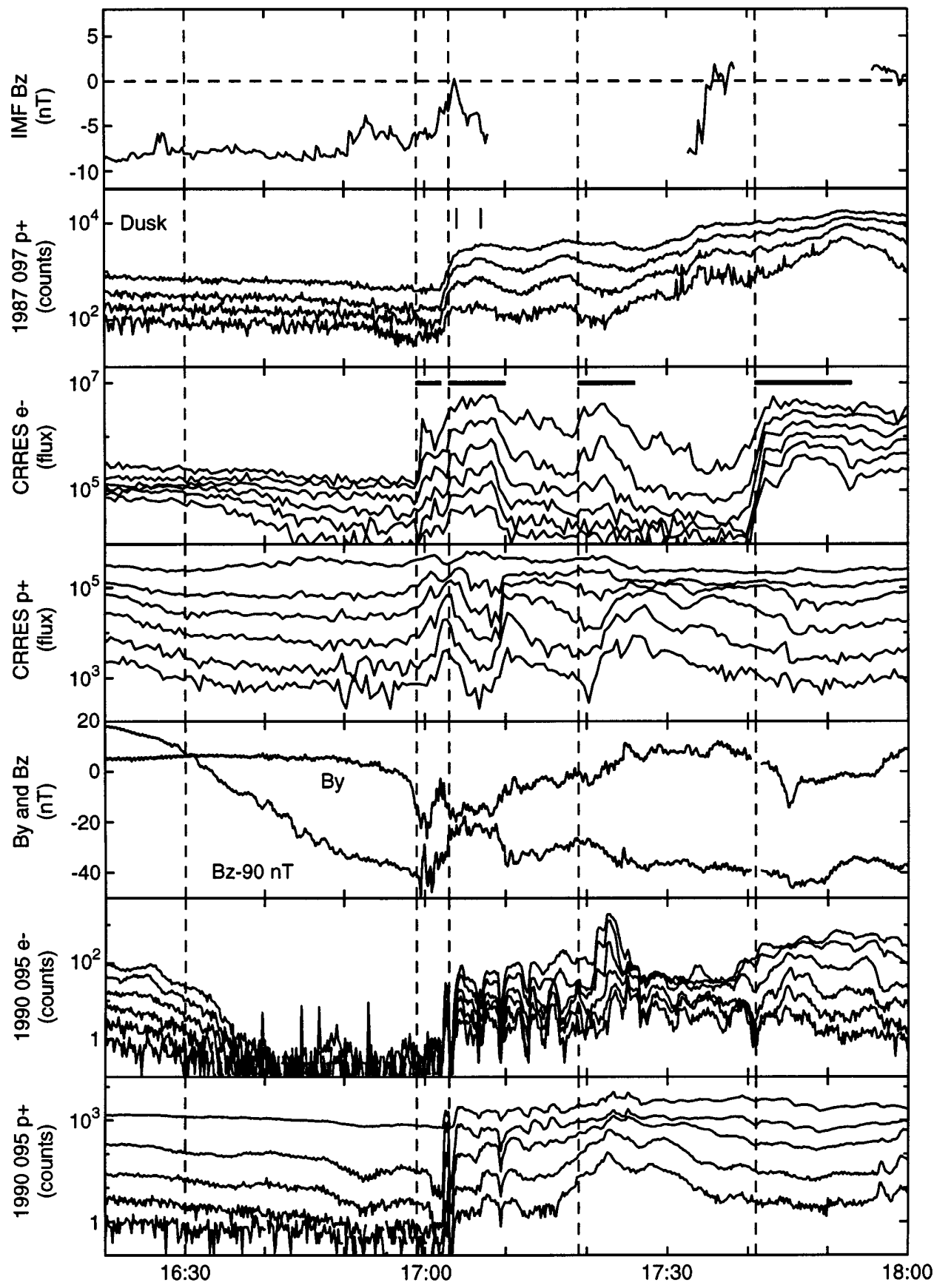

Fig. 3. IMP-8, CRRES and geosynchronous data. Panels from top to bottom are: 1 IMF $B_{z}(\mathrm{SE}) ; 21987$ 097 protons $(75-157 \mathrm{keV}) ; 3$ CRRES electrons (21.5-285 keV); 4 CRRES protons (37-3200 keV); 5 CRRES $B_{y}$ and $B_{z} ; 61990095$ electrons $(50-500$ $\mathrm{keV})$; and 71990095 protons $(50$ $400 \mathrm{keV}$ ). The CRRES fluxes are given in $\mathrm{cm}^{-2} \mathrm{~s}^{-1} \mathrm{sr}^{-1} \mathrm{keV}^{-1}$, while the geosynchronous data is given in counts only. The growth phase onset and the four intensifications as observed by CRRES are marked as dashed vertical lines than available in Fig. 3. The different panels contain $90^{\circ}$ PA electron and proton fluxes, $B_{y}$-components of the magnetic field, and the inclination of the field vector (angle between $B_{x}$ and $B_{z}$ ). In addition, Fig. 4 contains pitch-angle distribution (PAD) for the $21.5-31.5 \mathrm{keV}$ electrons. The intensification onset times are again shown as vertical dashed lines, and the particle energy ranges (in $\mathrm{keV}$ ) are listed on the right hand side of the corresponding panels. In the following we will discuss each electron intensification with detail in order to show that they can be divided into shorter time periods of increased fluxes, i.e. activations.

The first intensification was closely related to the substorm onset (Table 1). It consisted of two activations (labelled A1 and A2 in Fig. 4, panel 1), the former being strongest below $31.5 \mathrm{keV}$, the latter at somewhat higher energies (the different energy spectra are illustrated by the flux decrease at the lowermost energy bin during A2). However, the most notable particle feature during this period is the existence of almost field-aligned electrons at $<31.5 \mathrm{keV}$ (panel 3). In addition, the satellite observed strong signatures of field-aligned currents (panel 4) and a partial dipolarization that occurred during A2 (1701 UT, panel 5). The FAC signature started about one minute before the electrons were observed.

The second intensification showed the activations more clearly, being composed of three separate but similar electron activations (B1-B3 in Fig. 4). They had durations of about 1-2 min, a repetition rate of about 2 
$3 \mathrm{~min}$, and they were best observed at energies of 40 $81 \mathrm{keV}$. They were quite well seen also in other pitch angles (data not shown). The main magnetic field dipolarization occurred with B1.

There were at least three electron activations during the third intensification (C1-C3, panel 1 of Fig. 5); the last one may be composed of two separate activations. The duration and recurrence times of these activations were comparable to those of the second intensification, but the particle energies were lower $(<50 \mathrm{keV})$.

The 1990095 data support these observations (Fig. 3, two lowermost panels). For example, during and after the second intensification (i.e. at 1703-1717 UT), geosynchronous electron and proton fluxes showed periodicity that resembled the CRRES activations. On the other hand, there was no one-to-one correlation between the signatures at the two satellites, and the character of the modulation was different: one can see some indications of even smaller fine structure within the 1995095 intensity maxima, and the intensity minima were very pronounced, reaching the drop out level. During the third intensification, three or four activations can be recognized in the electron flux variations, obviously verifying the CRRES finding. Even during the first intensification there is a decrease of proton flux above $75 \mathrm{keV}$ (second energy channel) that coincided with $\mathrm{A} 2$, again verifying the existence of fine structure within the intensification.
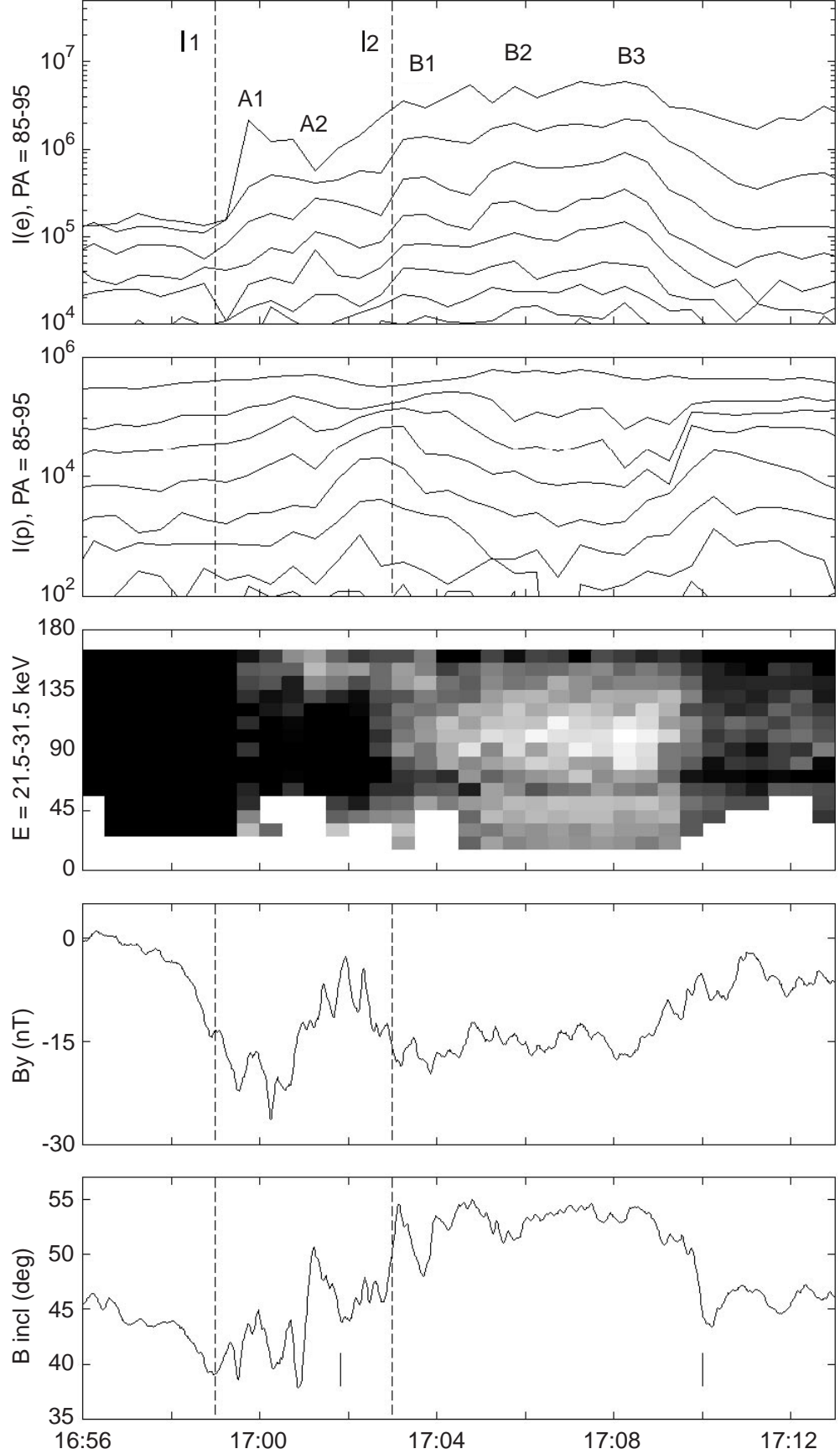

$21.5-31.5$

$31.5-40.0$

$40.0-49.5$

$49.5-59.0$

$59.0-69.0$

$69.0-81.0$

$81.0-94.5$

$94.5-112.0$

$37-54$

$54-69$

69-85

85-113

113-147

$147-193$

$193-254$

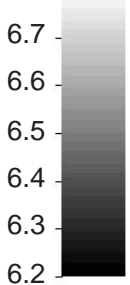

Fig. 4. The first two intensifications (about 1659-1702 UT and 1703-1710 UT, respectively). Shown are equatorial $\left(90^{\circ} \mathrm{PA}\right)$ electron (panel 1) and proton (panel 2) flux intensities at the lowermost energy channels, electron PAD at $21.5-31.5 \mathrm{keV}$ (panel 3), $B_{y}$ (panel 4), and inclination of $B$ (angle between $B_{x}$ and $B_{z}$, panel 5). Flux intensities are expressed in $\mathrm{cm}^{-2} \mathrm{~s}^{-1} \mathrm{sr}^{-1} \mathrm{keV}^{-1}$. The vertical tick marks in the lowermost panel indicate the intervals of increased field line stretching that coincide with the proton flux increases 


\subsection{Proton injections}

A repeating, intensification related pattern can be seen in the CRRES data (Figs. 3-5 and Table 1). In the three first intensifications, electron injection was followed by a proton injection that coincided in time with the end of the intensification. We will now investigate this further by calculating the particle and magnetic field energy densities at CRRES and tracing the observed protons back in time to their source regions.

Figure 6 (upper panel) presents the perpendicular energy density of protons and electrons and the magnetic field energy density during the event. Also here the dashed vertical lines are used to indicate the starting times of the growth phase and the intensifications, the bold horizontal lines showing also the durations of the latter. We can see that the electron energy density $W_{e}$, although peaking during the intensifications as it should, was of little importance for the total energy density, and that the magnetic energy density $W_{m}$ was generally dominant. However, the proton energy density $W_{p}$ was comparable to that of the magnetic field around the first three intensifications. To study this further, we noted that the first proton energy channel (37-54 keV) behaves often differently from the higher energy channels (see, for example, the second and third intensifications in Fig. 3 , panel 4). Accordingly, the proton energy densities were recalculated, now for different energy ranges. The lower panel of Fig. 6 presents ratios of both the 37$54 \mathrm{keV}$ differential energy density and the $>69 \mathrm{keV}$ integral energy density to the total energy density $W_{p}$. The relative importance of the low energy protons is obvious during the substorm growth phase and during all the intensifications. However, intervals of proton energy density increases were created also by the $>69 \mathrm{keV}$ protons, and they correlate very well with the three major high energy proton flux increases seen at the ends of the first three intensifications. There is thus a clear anti-correlation between the low and high energy proton fluxes, the former behaving more like the electron fluxes.

This difference between electrons and protons can be studied further. Figure 7 presents intensities from selected electron and proton energy channels as a function of $B_{z}$ during the growth phase (1610-1659 UT, the diamonds) and start of the active phase (16591708 UT, the pluses). Before the onset, both the electron and proton fluxes decreased generally together with the local magnetic field. After the onset of the substorm, however, the proton intensity variations were
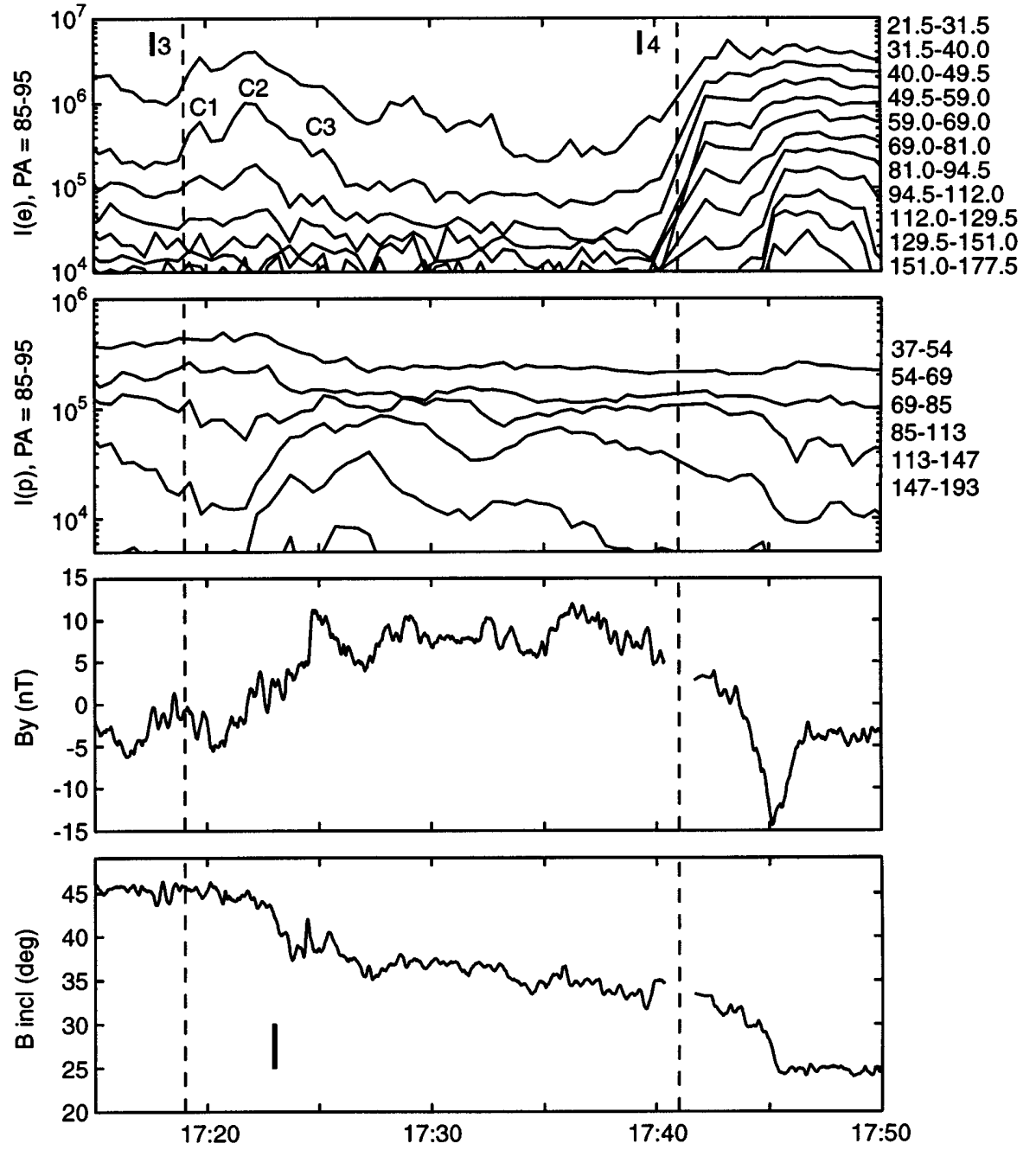

Fig. 5. The third and fourth intensifications. Shown are equatorial $\left(90^{\circ} \mathrm{PA}\right)$ electron ( panel 1) and proton (panel 2) flux intensities at the lowermost energy channels $B_{y}$ (panel 3 ), and inclination of $B$ (angle between $B_{x}$ and $B_{z}$, panel 4 ). Flux intensities are expressed in $\mathrm{cm}^{-2} \mathrm{~s}^{-1} \mathrm{sr}^{-1} \mathrm{keV}^{-1}$. The vertical tick mark in the lowermost panel indicate the interval of increased field line stretching that coincide with the proton flux increase 
independent of the magnetic field variations, whereas the electron flux variations were still ordered by $B_{z}$.

Because the high energy protons showed clear energy dispersion during the first (Fig. 4, panel 2) and the third intensification (Fig. 5, panel 2), we can also estimate when and where they were injected. Backward tracings, using the simple dipole model in Roederer (1970), showed that the protons were, in both cases, injected during the (CRRES electron) intensification they relate to, i.e. about 1701 and 1719 UT (see Table 1), and about $40 \mathrm{~min}$ and $90 \mathrm{~min}$ MLT eastward, respectively, from CRRES. The triangles on the $x$-axis of Fig. 1 show the approximate locations of these source regions (numbers referring to the intensifications).

Finally, note that the proton injections seem to correlate with the intervals of increased field line stretching, which are marked in the lowermost panels of Figs. 4 and 5 with vertical tick marks. For example, at the end of the first intensification, the dipolarization that had occurred during A2 was partly cancelled. Similar features can be seen during the second and third intensifications.

\section{Discussion}

In this study, we want to stress two important points seen in the case study presented: (1) fine structure in the activation time scale ( 2 min or less) can be seen in the injected electron fluxes and (partly) in the magnetic field fluctuations just as it has been observed in protons (Belian et al., 1984); (2) it is possible that high energy protons produced by substorm activity are also able to affect the way in which the activity develops further, and thus create some of the observed fine structure (at least on the time scale of intensifications, i.e., several minutes).

\subsection{Intensifications}

The substorm growth phase was characterized with a prolonged period of southward IMF $B_{z}$ component (Fig. 3, uppermost panel). The southward turning occurred at about $1606 \mathrm{UT}$, and taking into account the $\sim 6$ min time delay with the observed solar wind speed of $\sim 420 \mathrm{~km} / \mathrm{s}$, this corresponds well with the growth phase onset derived from ground, about 1610 UT. CRRES observed the corresponding changes at about 1630 UT, i.e., a little later. However, also the IMF $B_{z}$ reached its minimum, about $-9 \mathrm{nT}$, only at about 1615 UT (this is not seen in Fig. 3). Later on, the sharp decrease in the strength of the southward component at about 1651 UT fits very well with the first ground-based onset signatures at about 1657 UT, indicating that the substorm was triggered.

The substorm itself consisted of a series of electron intensifications seen both by CRRES and 1990 095. This activity was distributed over a sector several hours wide in MLT. We can use different data sets to investigate the details:
1. Observed differences between particle fluxes at CRRES and 1990 095, the latter being about 40-60 min MLT east of CRRES (Fig. 3).

2. Drift calculations on the dispersive ions at CRRES, indicating the azimuthal distance to the proton source (Figs. 4 and 5).

3 . The ground based magnetometer and ASC recordings (Fig. 2, Table 1).

The first intensification was observed only by CRRES, and the drift calculations on the dispersive ions indicated that they originated from about $40 \mathrm{~min}$ MLT away from CRRES (triangle 1 in Fig. 1). Therefore the position of the eastward edge of the source was not far from 1990 095. As the second intensification was seen both by CRRES and 1990 095, it extended further toward east. However, the lack of proton dispersion makes it difficult to make any estimations about this. The third intensification was also observed by both satellites, and on the ground it is best seen in the Dixon data. The dispersive ion source was calculated to be about $90 \mathrm{~min}$ east of CRRES (triangle 3 in Fig. 1). Note that the Cape Wellen data indicates strong activity peaking about 6-7 min later

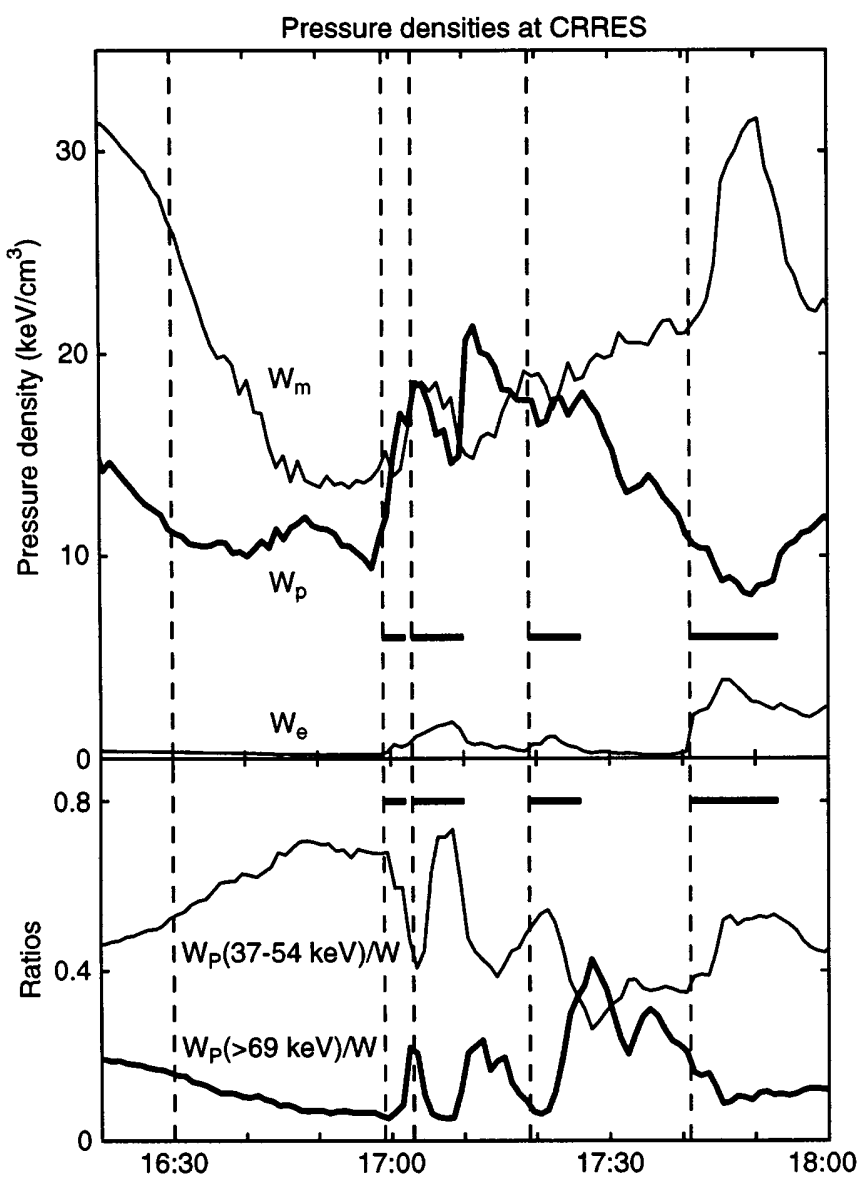

Fig. 6. Upper panel. Energy densities of the magnetic field $\left(W_{m}\right)$, perpendicular electrons $\left(W_{e}\right)$ and protons $\left(W_{p}\right)$ during the growth and active phases on February 9, 1991. Lower panel. Relative contribution of the $37-54 \mathrm{keV}$ protons and the $>69 \mathrm{keV}$ protons to total proton energy density $W$ 

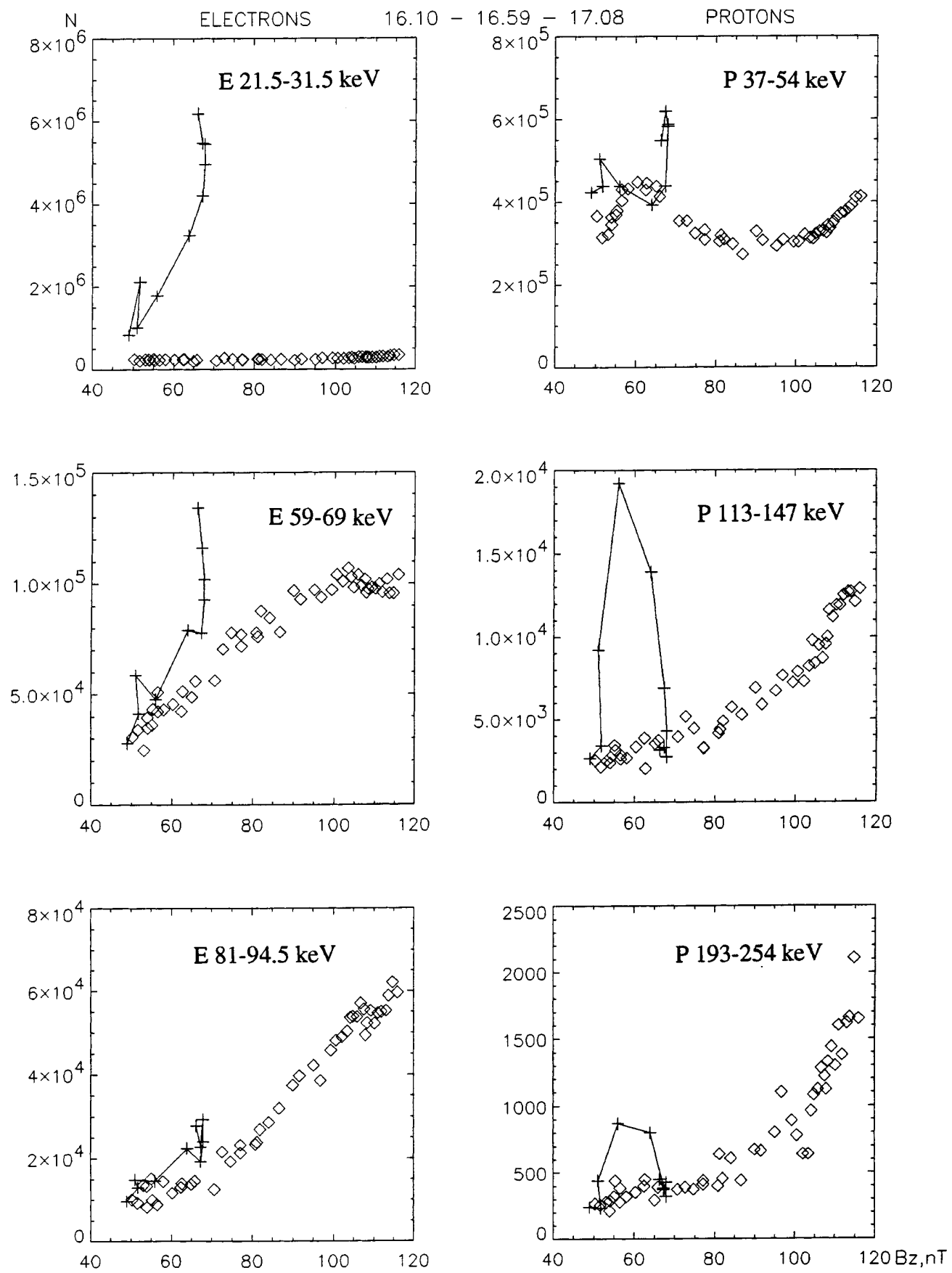

Fig. 7. The particle intensities during the growth phase (the diamonds) and the first minutes of the active phase (the pluses) as a function of $B_{2}$. Left column, trapped electrons; right column, protons. Units are in $\mathrm{cm}^{-2} \mathrm{~s}^{-1} \mathrm{sr}^{-1} \mathrm{keV}^{-1}$

eastward of CRRES. This is considered to be a separate intensification occurring in the dawn sector. The fourth CRRES intensification was registered close to Troms $\varnothing$ and Kilpisjärvi, the eastward edge being near the satellite (as derived from the weak electron dispersion and absence of protons). Thus, the azimuthal extension of an intensification can be rather limited or span a few hours in MLT. This is not surprising, as for example Belian et al. (1984) found longitudinal extensions of $45^{\circ}(3 \mathrm{~h})$. It is also obvious that the longitudinal location of the active region can change from one intensification to another. Therefore, an intensification can be defined as the longest interval of local substorm enhancement.
It has been shown that the substorm active phase often consists of two separate phases, active-convective and expansion (e.g. Lazutin et al., 1984; Mishin et al., 1992). During the active-convective phase the loading process is still strong and comparable with the unloading of energy, while during the expansion phase the unloading becomes the main process. The present event seem to consists of a active-convective phase during which intensification occur more independently of each other and the possible poleward excursions are restricted and localized. Only during the fourth intensification the global northward expansion commenced, as seen from the ground based data (Table 1). This view is backed up by the IMF data showing northward turning around 
the time of the last intensification (Fig. 3, uppermost panel).

The first intensification was different from the others, and we have reasons to believe that CRRES was closest to the activity centre, i.e., the formation of the substorm current wedge, during this time. This is supported by the (nearly) field-aligned $21.5-31.5 \mathrm{keV}$ electrons (Fig. 4, panel 3) as well as the field-aligned $\mathrm{keV}$ electrons (Johnstone et al., 1996) seen during this intensification. Satellite observations of the former, energetic electron fluxes at substorm onset are rather rare (Nielsen et al., 1982; Kremser et al., 1982, 1988), and ground-based measurements have shown that the precipitating particles are most energetic during the onset phase of the substorm (Olsson et al., 1996). However, note also that the first electron signatures are somewhat delayed from the first FAC and ground based signatures (Table 1), and that at about 1659-1701 UT the magnetic field shows precursory effects where it fluctuates without any lasting dipolarization effect (Fig. 4, panel 5).

One feature common to the three first intensifications is the partial return to more stretched field line configuration at or near the end of intensification. We will discuss this more later, in connection with the proton injections coinciding in time with these signatures.

\subsection{Activations}

The division of intensifications into separate activations was inferred from the CRRES electron data (panel 3 in Fig. 3, and panel 1 in Figs. 4 and 5). In addition, the 1990095 data supported this view, although we do not expect perfect correlation between the satellites within these time scales. Note also that the fact that the variations in the geosynchronous particle fluxes at 1703 1717 UT were of periodic drop out type is not a problem for our scheme, since also the drop outs must be related to injections that occur somewhere close. Thus, when the two satellites were within the same intensification source, they registered particle variations with comparable, but not the same activation structure. The lack of one-to-one correlation between individual activations could even be used to define an upper limit for their azimuthal extension (note that the difference in radial distance can also have an effect). The resulting one hour is comparable to the length of the individual activation arcs within a westward travelling surge (e.g., Murphree and Cogger, 1992; Nakamura et al., 1993). Since no high time resolution optical data from our event exist, we do not know how our activations/intensifications affect the auroral display along the CRRES field line. However, the observed field aligned electrons and the field aligned current signatures indicate that there is a connection, at least during the first intensification, between the observed activity in space and conjugate auroral ionosphere. This would be natural, as Yahnin et al. (1990) have shown that there is a connection between groundbased fine structure signatures and high energy proton injection fine structure first described by Belian et al. (1984).
The CRRES measurements during the first intensification show also how partial magnetic field dipolarizations and field-aligned current structures can occur at the time scales of activations (Fig. 4, panels 4 and 5). This indicates that the dipolarization process can be step-like, each step representing a localized activation. That similar signatures are not seen elsewhere may well be due to the fact that CRRES was closest to the activity centre during this time, as argued already.

To conclude this subsection, we note that substorm intensifications can be comprised of shorter elements that we call activations. The time scale of individual activations are of the order of 2 min which agrees, e.g., with the impulsive dissipation event (IDE) scenario proposed for substorm development (Sergeev et al., 1996). The present data set does not allow us to speculate further what this means in terms of substorm onset theories.

\subsection{Role of the protons}

Our results suggest that the injected high energy protons may play an important role in the intensifications. According to Figs. 4 and 5 (panels 2) and Fig. 6 (lower panel), the main $>69 \mathrm{keV}$ proton flux increases occurred at the end of the three first intensifications. In addition, they correlate with periods of local magnetic field line stretching (lowermost panels of Figs. 4 and 5). We suggest that these high energy protons give dynamic input that has important consequences, that they are in fact quenching, if only locally, the ongoing intensification, and are simultaneously creating favourable conditions for the fourth (last) intensification to occur. Note that we still think that the lower energy protons create the high level of particle energy density and the largescale enhancement of the cross tail current, and hence the word "dynamic" was used.

That the fourth intensification is not related to such a proton signature at CRRES is easily understood by the drift direction of the protons. It is obvious that, in order to observe the present events, the satellite must be situated in the western part of azimuthally extended acceleration region. This may not be always the case. However, we would like to note that the event presented here is not exceptional, and other similar cases are currently being studied using CRRES data.

Our scheme goes as follows (we will first make a more general statement and then point out, in parenthesis, how our observations support the claim).

1. Along with the formation of enhanced energetic proton flux during an intensification, we get a particle population that carries a relatively large amount of energy density with it. (Our drift calculations indicate that the protons are produced during the same intensifications as the electrons preceding them. We also calculated that the energy densities of the drifting proton clouds are comparable to the magnetic field energy densities. The electron energy densities are not comparable with these two values. 
This difference between the particles is also seen in Fig. 7.)

2. Thus, because of the increased cross-tail current and the diamagnetic effects of the ion cloud, the local magnetic field can be stretched tailward again. (Our data shows good correlation between the ion injections and periods of magnetic field stretching.)

3. This process is competing with the original, still ongoing substorm instability that created the injections in the first place, and resulted in field line dipolarization. (Note that we assume a situation where the source region is azimuthally extended, and the produced protons drift, at least partly, over this active region before leaving it. The particle signatures presented in this study support this view.)

4. If there are differences in the injection process for the electrons and protons, for example different azimuthal extents or locations, or if the higher energy ions drift differently from other particles, we may have time delays between the flux increases. (The time delay is very obvious in our data, and can be explained simply by drift from an eastern part of the intensification region during the first and the third intensification, as seen from the observed energy dispersion. The dispersionless proton injection during the second intensification must be explained in some other way.)

5. While within the active region, the protons dominate both the electron population and the magnetic field, with the result that the instability responsible for the intensification may be affected or even killed. (In this study, the arrival of protons correlate very well with the end of intensifications.)

6. At the same time, the protons could be creating a favourable condition for another intensification to occur at a different location in the direction of their drift. (The fourth intensification close to Kilpisjärvi could be formed this way. Protons were drifting towards 1987097 during the whole active period, see Fig. 3, panel 2, keeping the local field lines in a stretched configuration until the last intensification which started the main northward expansion of the substorm. That the intensification seems to be eventually triggered by a change in the IMF is not a contradiction.)

In addition to this quenching effect, note that the $<120 \mathrm{keV}$ protons may also have something to do with the onset of the first intensification, as their flux intensity started to increase just after the substorm onset, i.e. about two minutes before the first electrons were observed (Fig. 3, fourth panel, and Table 1).

It is possible that also the activation structures are controlled by the protons, although it is more difficult to prove from the observations. For example, CRRES proton data shows some indications of fine structure: see the short lived proton peak just before 1701 UT in Fig. 4, panel 2, and the two maxima in the proton fluxes relating to the third intensification in Fig. 5, panel 2 .

\section{Conclusion}

We have shown, using multisatellite, near-geosynchronous data, that magnetospheric substorms can consist of several intensifications, the dynamics of which may be controlled by bursts of high energy $(>69 \mathrm{keV})$ proton fluxes produced during the same intensifications. We suggest that the substorm instability responsible for the intensifications introduces a negative feedback loop into the system, creating this way the observed fine structure at the intensification time scales. In addition, each intensification can be subdivided into several short-lived activations, coinciding sometimes with separate partial dipolarizations.

Acknowledgements. L. L. L. wants to thank the Austrian Academy of Sciences for a fellowship and the Space Research Institute in Graz for providing working possibilities. The work of R. R. has been supported by the Academy of Finland through the Space Institute of Oulu. The authors are grateful to Tilmann Bösinger for fruitful discussions about the event during the course of the work and to Nelson Maynard for information about the CRRES electric field data.

Topical Editor K.-H. Glaßmeier thanks A. E. Bering and another referee for their help in evaluating this paper.

\section{References}

Anderson, K. A., Energetic electron fluxes in the tail of the geomagnetic field, J. Geophys. Res., 70, 4741-4763, 1995.

Angelopoulos, V., W. Baumjohann, C. F. Kennel, F. V. Coroniti, M. G. Kivelson, R. Pellat, R. J. Walker, H. Lühr, and G. Paschmann, Bursty bulk flows in the inner central plasma sheet, J. Geophys. Res., 97, 4027-4039, 1992.

Belian, R. D., D. N. Baker, E. W. Hones, P. R. Highbie, Highenergy proton drift echoes: multiple peak structure, J. Geophys. Res., 89, 9101-9106, 1984.

Bösinger, T., and A. G. Yahnin, Pi1B type magnetic pulsations as a high time resolution monitor of substorm development, Ann. Geophysicae, 5A, 231-238, 1987.

Bösinger, T., K. Alanko, J. Kangas, H. J. Opgenoorth, and W. Baumjohann, Correlation between Pi type magnetic micropulsations, auroras, and equivalent current structures during two isolated substorms, J. Atmos. Terr. Phys., 43, 933-945, 1981.

Grande, M., C. H. Perry, D. S. Hall, B. Wilken, S. Livi, F. Søraas, and J. F. Fennell, Composition signatures of substorm injections. Proc. International Substorm Conference (ICS-1), ESA SP-335, 485-490, 1992.

Johnstone, A. D., S. Szita, N. J. Flowers, and N. P. Meredith, Observations of particle injection from CRRES, Proc. Third International Substorm Conference, Versailles, France, ESA SP389, 467-471, 1996.

Kisabeth, J. L, and G. Rostoker, The expansive phase of magnetospheric substorms, 1, Development of the auroral electrojets and auroral arc configuration during a substorm, J. Geophys. Res., 79, 972-984, 1974.

Korth, A., G. Kremser, B. Wilken, W. Güttler, S. L. Ullaland, and R. Koga, Electron and proton wide-angle spectrometer (EPAS) on the CRRES spacecraft, J. Spacecraft, 29, 609-614, 1992.

Koskinen, H., R. E. Lopez, R. J. Pellinen, T. I. Pulkkinen, D. N. Baker, and T. Bösinger, Pseudo-breakup and substorm growth phase in the ionosphere and magnetosphere, J. Geophys. Res., 98, 5801-5813, 1993.

Kremser, G., J. Bjordal, L. P. Block, K. Brфnstad, M. Håvåg, I. B. Iversen, J. Kangas, A. Korth, M. M. Madsen, J. Niskanen, W. 
Riedler, J. Stadsnes, P. J. Tanskanen, K. Torkar, and S. L. Ullaland, Coordinated balloon-satellite observations of energetic particles at the onset of a magnetospheric substorm, J. Geophys. Res., 87, 4445-4453, 1982.

Kremser, G., Korth, S. L. Ullaland, S. Perraut, A. Roux, A. Pedersen, R. Schmidt, and P. J. Tanskanen, Field-aligned beams of energetic electrons $(16 \mathrm{keV} \leq E \leq 80 \mathrm{KeV})$ observed at geosynchronous orbit at substorm onsets, J. Geophys. Res., 93, 14453-14464, 1988.

Lazutin L., A. Khrustchinsky, G. Gustafsson, G. Kremser, A. Melnikov, W. Riedler, J. Sakharov, K. Torkar, and J-P. Treilhou, SAMBO-GEOS: Substorm trapped particle boundary movements, particle precipitation and acceleration, IMS Symp, COSPAR, Graz, Austria, 1984.

Lui, A. T. Y., R. E. Lopez, S. M. Krimigis, R. W. McEntire, L. J. Zanetti, and T. A. Potemra, A case study of magnetotail current sheet disruption and diversion, Geophys. Res. Lett., 15, 721-724, 1988.

Lui, A. T. Y., R. E. Lopez, B. J. Anderson, K. Takahashi, L. J. Zanetti, R. W. McEntire, T. A. Potemra, D. M. Klumpar, E. M. Greene, and R. Strangeway, Current disruptions in the nearEarth neutral sheet region, J. Geophys. Res., 97, 1461-1480, 1992.

Mauk, B. H., and C. E. McIlwain, Correlation of Kp with the substorm injected plasma boundary, J. Geophys. Res., 79, 31933196, 1974.

Maynard N. C., Burke W. J., Basinska E. M., G. M. Erickson, W. J. Hughes, H. J. Singer, A. G. Yahnin, D. A. Hardy, and F. S. Mozer, Dynamics of the inner magnetosphere near times of substorm onsets, J. Geophys. Res., 101, 7705-7736, 1996.

McIlwain, C. E., Substorm injection boundaries, in Magnetospheric Physics, Ed. B. M. McCormac, D. Reidel, Dordrecht-Holland/ Boston-USA, 143-154, 1974.

Mishin, V., J. Woch, L. Eliasson, T. Saifudinova, A. Bazarzhapov, D. Shirapov, and S. Lunyushkin, Substorm scenario with two active phases: a study of CDAW-9C events, Proc. First International Substorm Conference, Kiruna, Sweden, ESA SP-335, 383-389, 1992.

Murphree, J. S., and L. L. Cogger, Observation of substorm onset, Proc. International Conference on Substorms (ISC-1), ESA SP335, 207-212, 1992.

Murphree, J. S., and R. D. Elphinstone, Correlative studies using the VIKING imagery, Adv. Space Res., 8, (9)9-(9)19, 1988.

Nakamura, R., T. Oguti, T. Yamamoto, and S. Kokubun, Equatorward and poleward expansion of the auroras during auroral substorms, J. Geophys. Res., 98, 5743-5759, 1993.

Nielsen, E., A. Korth, G. Kremser, and F. Mariani, The electron pitch angle distribution at geosynchronous orbit associated with absorption spikes during the substorm expansion phase, J. Geophys. Res., 87, 887-894, 1982.

Ohtani, S., K. Takahashi, L. J. Zanetti, T. A. Potemra, R. W. McEntire, and T. Iijima, Initial signatures of magnetic field and energetic particle fluxes at tail reconfiguration: explosive growth phase, J. Geophys. Res., 97, 19311-1932, 1992.

Olsson, A., M. A. L. Persson, H. J. Opgenoorth, and S. Kirkwood, Particle precipitation in auroral breakups and westward traveling surges, J. Geophys. Res., 101, 24661-24673, 1996.
Pytte, T., H. Trefall, G. Kremser, P. J. Tanskanen, and W. Riedler, On the morphology of high-energy $(>30 \mathrm{keV})$ electron precipitation at the onset of negative magnetic bays, J. Atmos, Terr. Phys., 38, 757-773, 1976.

Rasinkangas, R., V. A. Sergeev, G. Kremser, T. Ulich, H. J. Singer, and A. Korth, Current disruption signatures at the substorm onset observed by CRRES, Proc. Second International Substorm Conference, Fairbanks, Alaska, Geophysical Institute, University of Alaska Fairbanks, 595-600, 1994.

Roederer, J. G., Dynamics of geomagnetically trapped radiation, Springer-Verlag Berlin Heidelberg New York, 1970.

Rostoker, G., S.-I. Akasofu, J. Foster, R. A. Greenwald, Y. Kamide, K. Kawasaki, A. T. Y. Lui, R. L. McPherron, and C. T. Russell, Magnetospheric substorms - definition and signatures, J. Geophys. Res., 85, 1663-1668, 1980.

Rostoker, G., S.-I. Akasofu, W. Baumjohann, Y. Kamide, and R. L. McPherron, The roles of direct input of energy from the solar wind and unloading of stored magnetotail energy in driving magnetospheric substorms, Space Sci. Rev., 46, 93-111, 1987.

Sergeev, V. A., T. Bösinger, and A. T. Y. Lui, Impulsive processes in the magnetotail during substorm expansion, J. Geophys., 60, 175-185, 1986a.

Sergeev, V. A., R. J. Pellinen, T. Bösinger, W. Baumjohann, P. Stauning, and A. T. Y. Lui, Spatial and temporal characteristics of impulsive structure of magnetospheric substorms, J. Geophys., 60, 186-198, 1986b.

Sergeev, V. A., T. I. Pulkkinen, and R. J. Pellinen, Coupled-mode scenario for the magnetospheric dynamics, J. Geophys. Res., 101, 13047-13065, 1996.

Sergeev, V. A., M. A. Shukhtina, R. Rasinkangas, A. Korth, G. D. Reeves, H. J. Singer, M. F. Thomsen, and L. I. Vagina, Event study of deep energetic particle injections during substorm, accepted by J. Geophys. Res., 1998.

Singer, H. J., W. P. Sullivan, P. Anderson, F. Mozer, P. Harvey, J. Wygant, and W. McNeil, Fluxgate magnetometer instrument on the CRRES, J. Spacecraft, 29, 599-601, 1992.

Shepard, G. G., and M. S. Murphree, Diagnosis of auroral dynamics using global auroral imaging with emphasis on localized and transient features, in Auroral physics, Eds. C.-I. Meng, M. J. Rycroft and L. A. Frank, Cambridge University Press, Cambridge, UK, pp. 289-298, 1990.

Williams, P. J. S., T. S. Virdi, S. W. H. Cowley, and M. Lester, Shortlived bursts of plasma velocity in the auroral zone. 1. Observational evidence from radar measurements, J. Atmos. Terr. Phys., 52, 421-430, 1990.

Yahnin, A. G., V. A. Sergeev, R. J. Pellinen, W. Baumjohann, K. U. Kaila, H. Ranta, J. Kangas, and O. M. Raspopov, Substorm time sequence and microstructure on 11 November 1976, J. Geophys., 53, 182-197, 1983.

Yahnin, A. G., T. Bösinger, J. Kangas, and R. D. Belian, Some implications on substorm dynamics inferred from correlations between multiple flux peaks of drifting high-energy proton clouds and ground observations, Ann. Geophysicae, 8, 327-336, 1990. 